\title{
Strategy for IP traffic support with QoS in OBS networks
}

\author{
Xavier Hesselbach, Member, IEEE, Mónica Huerta* and Rodolfo Alvizu* \\ Technical University of Catalonia, UPC, Barcelona, Spain. \\ * Universidad Simón Bolivar, USB, Caracas, Venezuela. \\ Tel: + 3493 4015987, Fax: + 3493401 1058, email: xavierh@entel.upc.edu
}

\begin{abstract}
This paper presents a new strategy for burst reservation in OBS networks for the transport of IP/DiffServ traffics with Quality of Service (QoS) constraints using MPLS. The current resource reservation protocols research in OBS offer several algorithms for burst transmission, some of them taking into account QoS parameters. In this paper, these solutions will be shown according to the needs of IP/DiffServ/MPLS traffic. The main features of each strategy will be discussed and an enhanced algorithm proposed.
\end{abstract}

Keywords: Services classification, self-configuring, OBS, QoS, MPLS, DiffServ.

\section{INTRODUCTION}

The IP traffic is the core in the most recent applications delivered in Internet. These new applications demands more bandwidth and controlled delay in the network. For example, video contents become widely deployed and emerge as the killer application for the networks of the future where self-organizing mechanisms should be provided to guarantee the requirements, even in case of network failure or dynamic bandwidth allocation in the links. Each single stream can consume from $1 \mathrm{Mbps}$ to $100 \mathrm{Mbps}$, so the aggregation of video traffics coming from millions of users can overload a traditional core network. Therefore, the new generation networks should provide QoS, Quality of Service, (meaning bandwidth, delay, jitter and losses) according to the needs of each user and the agreement with the network provider. The key problem is how can converge the problem of high speed networks with services classification and resources discovery. In this paper, we propose a strategy where the traffic is marked to belong to a certain class of traffic or priority and, later, the OBS (optical burst switching) technology will transmit every burst according to this mark.

In recent years there has been active research in the field of multiprotocol label switching (MPLS). Multi Protocol Label Switching (MPLS) is a flexible routing mechanism based on the assignment of flows to complete end-to-end paths within an Autonomous Domain. "Traffic engineering" in is one of the primary applications envisioned for MPLS. This paper presents the problem of integrate IP with Differentiated Services traffic transmitted over MPLS and an OBS network and suggests a strategy for QoS provision. The paper is organized as follows. In the next section, a quick overview of QoS in IP using DiffServ and MPLS networks is presented, with a short taxonomy of QoS in OBS. The section 3 shows how OBS can transport MPLS traffic. The next section focuses on a proposal for provisioning QoS on OBS. The section 5 presents the existing signaling protocols for the reservation policy for OBS networks and the arguments for and against. The final section summarizes and concludes this paper.

\section{QOS IN IP/DIFFSERV/MPLS AND IN OBS NETWORKS}

The network services can be classified according to their level of QoS constrains, which describes how tightly the service can be bound by specific bandwidth, delay, jitter, and loss characteristics. At the first sight, three basic levels of end to end QoS can be provided across a heterogeneous network: The best-effort service, differentiated service (also called soft QoS), and guaranteed service (also called hard QoS), where an absolute reservation of network resources for specific traffic is provided through bandwidth reservation mechanisms. The MPLS shim header of the packets as a criteria to allocate different amount of resources for each Class of Service $(\mathrm{CoS})$ can be used. In this scenario, MPLS can support a degree of QoS by means of the FEC (Forwarding Equivalent Class).

In OBS, several signaling schemes have been proposed to support QoS. The basic problem is to determine the criteria for choosing a signaling method. The selection can be made according to several parameters, such as complexity of the algorithm, optimization, etc, but what the user applications needs is the guarantee for QoS, that means bandwidth, delay bounds, jitter and losses. This is what an OBS network should provide to an IP/DiffServ/MPLS protocol stack in such a way that can be managed an organized by upper layers in order to allocate classes of service according to existing standards for MPLS, DiffServ and IP traffic.

The main scheme for controlling (or administrate) the QoS in OBS is the control of the offset, in order to isolate classes of bursts, such as high and low priority bursts. In this kind of methods, an extra offset is given to the higher priority bursts, to reserve resources further in advance the arrival of the burst and so increasing the probability of a successful reservation. One limitation of the offset schemes is that scheduled low priority bursts can result in the loss of arriving high priority bursts. Therefore, other approaches for provision of QoS are needed. Some works are nowadays discussed, based on priorization of the contention resolution. These algorithms are not discussed in this paper and left for a future work. 


\section{OPTICAL BURST SWITCHING USING MPLS}

Semi-permanent data pipes can be set up between different ingress-egress router pairs using an MPLS-type technique. MPLS uses labels to make forwarding decisions at the network nodes. In MPLS, the space of all possible forwarding options is partitioned into forwarding equivalence classes (FECs). For example, all the packets destined for a given egress and having the same quality of service (QoS) may belong to the same FEC. Each of the intermediate nodes uses the label of the incoming packet to determine its next hop, and also performs label swapping. MPLS can play a major role in traffic engineering and improving the throughput performance of an OBS-based network, as seen below. Each cross-connect in the optical backbone will have label swapping information about the pre-computed routes in its label information base (LIB). A LIB can be set up using standard techniques such as routing protocols with traffic engineering extensions to distribute information about the optical domain (available bandwidth per wavelength, number of wavelengths per fiber) and Constraint-Based Routing Label Distribution Protocol (CR-LDP) or Resource Reservation Protocol (RSVP) to distribute labels. Whenever an ingress router has a data burst to transmit, it refers to its LIB to determine the appropriate label. This label is included in the control packet that precedes this data burst. When the control packet arrives at any of the intermediate nodes, the following actions take place:

- The label in the control packet is used to point to the data burst forwarding information in the LIB such as the output interface and any priority or QoS information.

- The cross-connect is set up to switch the data burst corresponding to that control packet in the all-optical domain. For this, information in the control packet about the length and offset of the data burst is used in addition to the forwarding information derived from the LIB. In particular, the latter is used to determine the mapping from the incoming fiber and wavelength to the outgoing fiber and wavelength.

In order to be able to forward successive data bursts of the same connection (LSP) on different wavelengths in a given fiber, we propose that the label only specify incoming-fiber-to-outgoing- fiber mapping, while the information about the wavelength be appended to the outgoing label at every hop. The LIB may furnish other QoS information as well on the outgoing fiber, determining the eligibility of that data burst to use wavelength conversion, stating whether (in case of contention) the control packet is allowed to preempt some reservation already acquired by the control packet of low priority data burst, and so on.

- The control packet then undergoes label swapping (and wavelength information appending) and is forwarded on the dedicated control channel of the outgoing fiber as indicated by the LIB.

A functional diagram of an optical cross-connect supporting OBS and using MPLS-type forwarding is shown in Fig. 1.

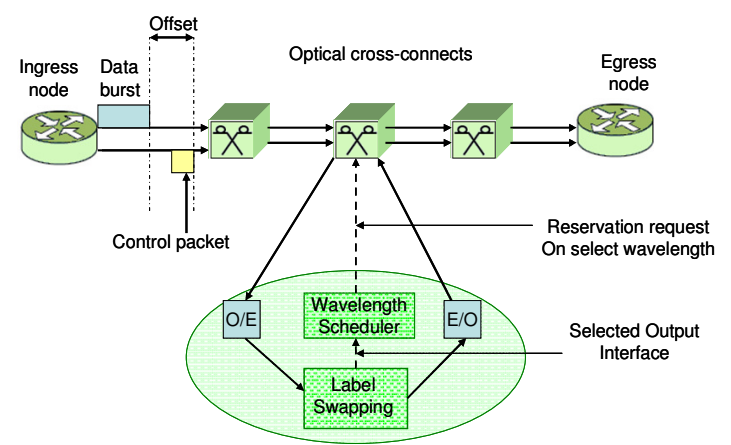

Fig. 1: A functional diagram of an optical cross-connect supporting OBS and using MPLS-type forwarding.

\section{A QOS ALGORITHM PROPOSAL FOR OBS}

A number of approaches for QoS provisioning in OBS networks have been proposed in the literature. These approaches can be classified into offset-based [1], strict priority [2], segmentation-based [3] or proportional QoS [4]. The main aim of the aforementioned proposals is to provide relative service differentiation with regards to packet loss probability. The use of classical fair scheduling algorithms in the data plane of optical nodes has generally been avoided in the literature. This is due to the absence of the concept of "packet queues" in optical nodes, beyond the number of packets that can be buffered (while in-flight) in Fiber Delay Lines.

Packets arriving at the ingress node are classified into one of the FECs already defined by the network operator. These packets are then shaped and policed to conform to the QoS requirements of their FEC. Packets are then assembled into bursts in the burst assembly queue. The burst assembly process is carried out using the containerization with aggregation-timeout (CAT) algorithm. In principle, the function of the CAT algorithm is to assemble the arriving packets into data bursts, such that each data burst contains packets that belong to one FEC. Two parameters control this burst assembly process in CAT, namely the maximum burst size (Bmax) and burst assembly timeout (Tmax). The maximum burst size controls the maximum number of packets that can be in the same burst. If the incoming traffic intensity is high enough, the maximum burst size will be reached in a 
relatively short time. If, on the other hand, the traffic has a low arrival rate, the burst being assembled might have to be queued for a relatively long time until the maximum burst size is reached. In order to avoid large queuing delays for bursts, the assembly timeout parameter is used to release the burst under assembly. Thereafter, data bursts should be inserted into the bursts queue and scheduled for processing by the reservation manager according to their QoS requirements.

\section{SIGNALING PROTOCOLS}

Considering that the modes currently under investigation for the implementation of the next generation coarse packet switched optical networks, e.g. Burst Switching, are based on these reservation policies, in this section we present the resources reservations protocols for OBS.

\subsection{Tell And-Go (TAG)}

In this strategy, the burst is sent from an ingress node even if the establishment of an optical virtual path has not been completed. The burst follows the virtual path in parallel with the setup phase. The data burst and the setup message will be spaced by a guard time. This time allows the optical nodes to be set before the burst arrive. When the burst arrive to the egress node, an acknowledgment will be sent back to the source, and send out the set up message and the burst again. If the requested bandwidth cannot be granted at an intermediate node, the burst is not lost, and will be sent after a back-off time, but this aims to transmit some bursts several times. So, this protocol does not allow QoS or service differentiation. It is illustrated in Fig. 2 left.

\subsection{Tell And-Wait (TAW)}

In TAW the burst is emitted by an ingress node only if an optical virtual path has been set through the network up to the egress node, by sending a short request message and "waiting" for the acknowledge (ACK) message. When an intermediate node receives this message will make a reservation off a free data wavelength on the routed output; if the requested bandwidth is successfully reserved on all the links along the path, the ACK will be sent back to the source after the round-trip delay, and send out the burst immediately; Otherwise, a negative acknowledgment (NACK) will return to release the previously reserved bandwidth, and the source will have to try to make another request after a back-off time. If the request bandwidth cannot be granted at an intermediate node, the resource at the previous nodes will be wasted until the release messages arrive, but the burst is not lost, and will be sent after a back-off time. So, the utilization of resources is not efficient because reserved links last longer than the burst duration. It is shown in Fig. 2 right.
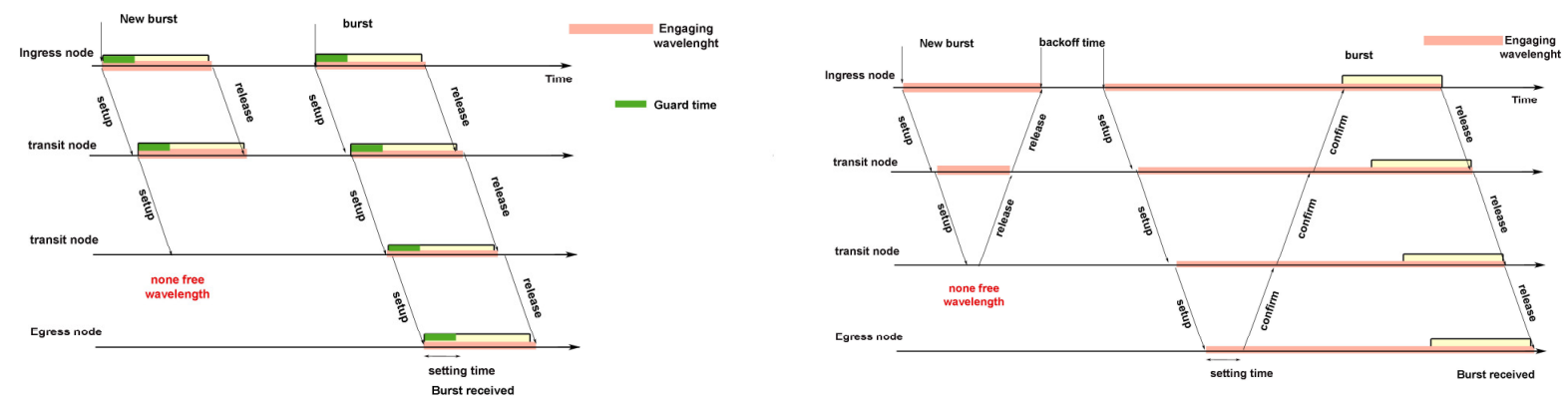

Figure 2 Tell And-Go (TAG) and Tell-And-Wait (TAW)

\subsection{Just-In-Time (JIT)}

In JIT, a burst transmission request is sent to a central scheduler. The scheduler then informs each requesting node the exact time to transmit the data burst, at the appropriate time, the source transmits its burst and intermediate switches are set "just in time", for an efficiently use of wavelength channels that are set up only for the required burst transmission time. Centralized protocols are neither scalable nor robust, a distributed control scheme would be preferred; however, such a scheme relies on synchronization and fast distribution of information on the state of the network, [6] provided a distributed version of JIT called Reservation with Just-InTime (RIT), which requires a copy of the request to be sent to all switches.

\subsection{Just-Enough-Time (JET)}

In JET, a setup message is emitted by an ingress node and after an extra offset time the burst is emitted, this time takes into account the delays experienced by the setup message within a node. The header carry the data burst's length, destination and arrival time, this allows to reserves all resources exactly for the transmission time of the burst, is called a reserve-a-fixed duration (RFD) scheme where the node can make delayed reservation of the capacity needed at the corresponding output port. JET is the most prevailing distributed protocol for OBS networks today that does not require any kind of optical delays. [5] Proposed a new approach that assigns different extra offset times to different service classes so as to provide differentiated services in terms of burst 
loss probability for classes of different priorities, this reduce the loss rate of high priority traffic at the expense of an increase in the loos rate of lower priority traffic. In the end-to-end delay, is present the extra offset time. It is shown in Fig. 3 right. Other problem is that high priority burst with large offset times will break the resource's free periods into small pieces, and only the burst with smaller size will have more probability to find a free wavelength. JET does not use an ACK for guaranteed the end-to-end lightpath, this cause loss of the bursts that have to be retransmitted for an upper layer.

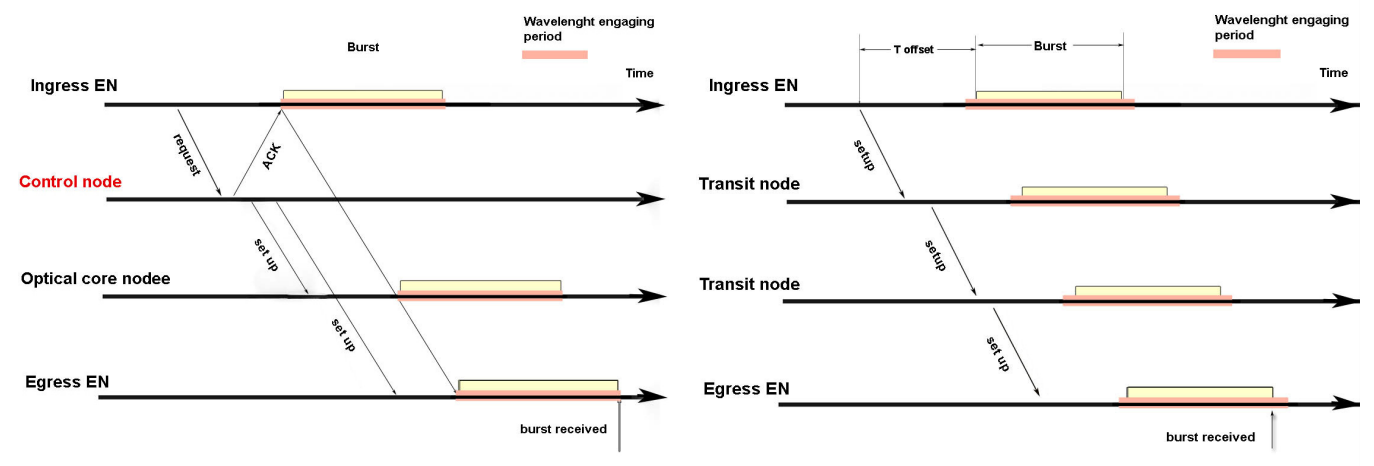

Figure 3 Just-In-Time (JIT) and Just-Enough-Time (JET)

Table 1. Signaling Protocols summary.

\begin{tabular}{|c|c|c|c|c|}
\hline Mechanism & Use resource & End-to-End delay & Resource reserve & DiffServ \\
\hline TAG & Efficient & Short & Guaranteed & Not supported \\
\hline TAW & Deficient & Long & Guaranteed & Not supported \\
\hline JIT & Efficient & Medium & Guaranteed & Supported \\
\hline RIT & Efficient & Medium & Guaranteed & Supported \\
\hline JET & Efficient & Short & No guaranteed & Supported \\
\hline
\end{tabular}

This table summarizes the general behavior of each signaling protocol according to the requirements of the traffic to be transported. From this, JET is the candidate for networks where delay is the key constraint, but resource reserves are not guaranteed. Therefore, it can be useless for constant bit rate traffics with real time requirements. In this case, JIT or RIT can fulfil this constraints but the end-to-end delay is larger than in JET. We conclude that a new signaling protocol is required taking into account the parameters for the control packet management in OBS. We suggest the inclusion of statistical per node behaviour of the network to route the bursts. It should be studied in a future work.

\section{CONCLUSIONS}

In this paper, an overview of signaling protocols for OBS networks has been presented in order to support IP/DiffServ/MPLS with QoS traffic. The protocols do not fulfil the requirements coming from the upper layers. Some work should still be done, and we suggest the inclusion of statistical per node behaviour. For future work: First is the study of mapping of classes from DiffServ to OBS. Second is the design of a control plane to manage the allocated traffic. Third is the analysis considering priorization of the contention resolution strategies for QoS.

\section{ACKNOWLEDGEMENTS}

This work has been partially supported by the national Spanish project CICYT TSI2007-66637-C02 and the European NoE Euro-NF 2008-2010.

\section{REFERENCES}

[1] M. Yoo, C. Qiao, and S. Dixit, "Optical burst switching for service differentiation in the next generation optical internet," IEEE Communications Magazine, pp. 98-104, February 2001.

[2] A. Kaheel and H. Alnuweiri, "A strict priority scheme for QoS provisioning in optical burst switching networks," in IEEE Symposium on Computers and Communications-ISCC'03. Turkey, 2003.

[3] V. M. Vokkarane and J. Jue, "Prioritized routing and burst segmentation for QoS in optical burst switched networks," in Optical Fiber Communication Conference- OFC'02. Anaheim, March 2002.

[4] Y. Chen, M. Hamdi, and D. Tsang, "Proportional QoS over OBS networks," in IEEE Global Telecommunications Conference- Globecom. San Antonio, November 2001.

[5] I. Widiaja,"Perfomance Analysis of Burst Admission-control Protocols," IEE Proc.Comm.,vol.142, 1995.

[6] G.C.Hudek, D.J.Muder, "Signalling Analysis for a Multi-Switch All-Optical Network," IEEE ICC, 1995. 